\title{
Diseño de un Sistema de Gestión Ambiental bajo la norma iso 14001:2015 en la mina de arcilla Sabanilla en San Juan del Cesar, La Guajira
}

\section{Resumen}

Danny López', Dayanis Cataño2, Keity Daza³, Miguel Llanes ${ }^{4}$

En esta investigación se implementó un diseño de Sistema de Gestión Ambiental para la mina de arcilla Sabanilla del municipio San Juan del Cesar, La Guajira, con base en los lineamientos establecidos por la norma internacional ISO 14001:2015, esto con el fin de mejorar el desempeño ambiental de la unidad minera que desde hace ya varios años viene extrayendo dicho recurso natural sin realizar gestión ambiental alguna, repercutiendo directamente en el ambiente y los diferentes componentes que lo conforman. Para esto se procedió analizar el contexto de la organización de la mina y establecer políticas ambientales para así proceder a la planificación de las acciones a desarrollar frente al manejo de los impactos encontrados en la mina. Luego se formuló una estrategia para la implementación y operación del Sistema de Gestión Ambiental (SGA), en el que se establecieron procedimientos para mejorar continuamente la eficacia del desempeño ambiental de la mina. Así mismo, el análisis realizado arrojó como resultado que la mina no lleva a cabo ningún tipo de gestión ambiental. En este sentido, fue posible identificar las partes interesadas y sus necesidades y expectativas respeto a la mina; logramos determinar que las principales son la comunidad, los empleados y los clientes. Y, además, que el SGA abarcara todos y cada uno de los componentes que forman parte de la red de procesos de la unidad minera, considerando también la totalidad de productos y actividades inmersas en esta.

\footnotetext{
IIngeniero en minas, doctor en Ciencias Gerenciales, docente del programa Ingeniería Ambiental de la Universidad de La Guajira. Pertenece al Grupo de Investigación IPAITUG. Correo: dlopezjđanniguajira.edu.co.

2Estudiante del programa Ingeniería Ambiental de la Universidad de La Guajira.

${ }^{3}$ Estudiante del programa Ingeniería Ambiental de la Universidad de La Guajira.

${ }^{4}$ Estudiante del programa Ingeniería Ambiental de la Universidad de La Guajira.
} 
Palabras clave: sistema de gestión ambiental, norma ISO14 001: 2015, política ambiental, contaminación atmosférica, residuos sólidos, escombros, suelos, fauna y flora, seguridad y salud en el trabajo y contratación de mano de obra.

\section{Introducción}

La minería se define como la ciencia, las técnicas y las actividades que tienen que ver con el descubrimiento y la explotación de yacimientos minerales. De manera estricta, el término se relaciona con los trabajos subterráneos o a cielo abierto. En la práctica, el término incluye las operaciones a cielo abierto, canteras, dragado aluvial y operaciones combinadas que incluyen el tratamiento y la transformación. La minería es una de las actividades más antiguas, la cual consiste en la obtención selectiva de minerales y otros materiales. (Ministerio de Minas y Energía, 2003).

Las formas de asentamiento del ser humano definen la ocupación del espacio y el uso de los recursos como el agua, la flora y la fauna el crecimiento poblacional ha influido significativamente en el cambio del uso del suelo y por consiguiente han aumentado los conflictos por el uso de las tierras, los cuales son resultado de la discrepancia entre el uso que el hombre hace del medio natural y aquel que debería tener, de acuerdo con la oferta ambiental. (Sánchez, 2001)

Debido a lo anterior, se propone diseñar un Sistema de Gestión Ambiental bajo los criterios de la norma ISO 14001 en la minería de arcilla.

Ahora bien,

un Sistema de Gestión Ambiental (SGA) es la parte del sistema general de gestión de calidad que incluye la estructura organizativa, las actividades de planificación, las responsabilidades, las prácticas, los procedimientos, los procesos y los recursos para desarrollar, aplicar, alcanzar, revisar y mantener la política ambiental. (Gaviria, 2007)

La realidad social, política, cultural y económica son aspectos determinantes para definir el entorno, el estilo y la calidad de vida que tenemos. El desenfreno de la sociedad consumista, el desarrollo tecnológico y el aumento de ingresos económicos ha dado lugar a la creación 
de innumerables procesos que además de "facilitarnos la vida" se están encargando de causar daños ecológicos que, sin duda alguna, ya se están manifestando en nuestras vidas. Dentro de estos es preciso mencionar la minería, que en las últimas décadas se ha convertido en una de las actividades económicas más promisorias a nivel mundial (Cardona, 2005).

Los productos minerales son consumidos preferentemente por los sectores industriales y los de construcción. Es por esto que podríamos argumentar, que en esta sociedad moderna a la que estamos insertos, la utilización de los recursos minerales y por consecuencia la actividad minera, es un sustento importante para la misma. (Villamizar, 2016)

En el caso colombiano, se llega a determina que "Colombia no es un país minero, ya que solo el $2,3 \%$ del producto interno bruto del país corresponde al procedente de dicha actividad". Pese a esto, para nadie es un secreto que el sector minero en los últimos años se ha consolidado como uno de los sectores de mayor interés en la economía del país, y que la producción de la mayoría de los minerales que se explotan ha presentado un aumento considerable, sobre todo en algunos departamentos, dentro de los que se destaca La Guajira (Acosta, 2014).

Uno de los principales problemas que afronta el sector minero en el departamento, es la minería ilegal; en la cual no existe gestión ambiental alguna, priorizando temas financieros, en muchos casos de sustento básico, desarrollando acciones eco-irracionales que no producen beneficios de fondo para la sociedad, convirtiéndose en un verdadero cáncer para los intereses nacionales, desde la óptica en que se le mire. (López, 2011)

Por su parte, el municipio San Juan del Cesar, La Guajira, no es ajeno a esta situación, dentro de su territorio se encuentra una mina artesanal de arcilla, en la que se explota el mineral de manera ilegal, ya que no cuenta con ningún tipo de autorización, licencia ambiental, contrato de concesión ni los respectivos permisos ambientales para el aprovechamiento de recursos naturales.

La norma ISO 14001: es una norma internacional de aplicación voluntaria, que establece los requisitos legales que debe cumplir una organización para gestionar la prevención 
de la contaminación y el control de las actividades, productos y procesos que causan o podrían causar impactos sobre el medio ambiente, $\mathrm{y}$ además, para demostrar su coherencia en cuanto al cumplimiento de su compromiso fundamental y respecto por el medio ambiente. (Icontec, 2005)

Sistema de Gestión Ambiental: se define como un sistema estructurado de gestión que incluye la estructura organizativa, la planificación de las actividades, las responsabilidades, las prácticas, los procesos, los procedimientos y los recursos para desarrollar, implantar, llevar a efecto, revisar y mantener al día los compromisos en materia de protección ambiental que suscribe una empresa. (Ministerio de Fomento, Industria y Comercio [MIFIC], 2015).

Dado que se quiso implementar un sistema que ayudara a la restauración de la mina, se miró cuáles son los problemas ambientales que esta le genera al medio ambiente para así poder mitigarlos.

A raíz de que para la fabricación de los bloques se necesita material vegetal, los mineros han llevado esto a un grado de destrucción del hábitat nativo de
Sabanilla, lo que con lleva a la desaparición de la fauna silvestre en varias zonas de la mina. Al hacer la remoción de la capa del suelo para la extracción de la arcilla, esos suelos quedan debilitados por los tipos de manejo que le dan. Otra problemática que es de gran importancia es toda la contaminación que es emitida por los hornos artesanales a la atmosfera, ya que estas partículas que generan pueden causar grandes enfermedades a los trabajadores de la mina.

También se pudo determinar que al ser extraída la arcilla se contamina gran parte del recurso hídrico del que ellos se benefician. No cuentan con un manejo de los residuos sólidos; todo lo que generan lo tiran, afectando el medio ambiente y esto, a su vez, les puede generar enfermedades a los trabajadores

\section{Materiales y métodos}

Según Hernández (2014),

con los estudios descriptivos se busca especificar las propiedades, las características y los perfiles de personas, grupos, comunidades, procesos, objetos o cualquier otro fenómeno que se someta a un análisis. Es decir, únicamente pretenden medir o recoger información de manera 
independiente o conjunta sobre los conceptos o las variables a las que se refieren.

Con base en lo anteriormente dicho, es preciso afirmar que la investigación es de este tipo porque uno de sus principales objetivos es describir las características, los impactos ambientales más relevantes que se generan en la zona que abarca la unidad minera y las actividades y procesos realizados en esta para, posteriormente, analizar la información obtenida y, de esta manera, implementar un diseño de gestión ambiental bajo la norma ISO 14001 de 2015 en la mina de arcilla sabanilla de San Juan del Cesar, Guajira.

En esta investigación también se implementa el método de tipo deductivo, dado que se parte de lo general, la aplicación de la norma internacional ISO 14001:2015, a lo particular, que en este caso es el diseño de un sistema de gestión ambiental para la mina de arcilla Sabanilla. El diseño se considera dentro de esta clasificación porque, para esta investigación, no es necesario realizar procedimientos que cumplan con las condiciones de un experimento. Además, teniendo en cuenta que para la realización de este estudio se busca, en primera instancia, observar el estado de cada uno de los componentes bióticos, abióticos y socioeconómicos del área que conforma la mina y el grado de alteración que estos presentan debido a la actividad productiva de la misma, es preciso afirmar que se trata de una investigación no experimental, puesto que para esto se observara el fenómeno tal como se da en su contexto natural para, posteriormente, ser analizado y por medio del SGA buscar posibles soluciones al mismo. Sobre todo, teniendo en cuenta que para tales fines no es necesario ningún tipo de manipulación de las variables por parte de los investigadores.

Para la realización de esta investigación y lograr los objetivos planteados, se establece el siguiente diseño de investigación, en el cual se empleó la metodología propuesta por la norma ISO 14001 de 2015 (Sistemas de Gestión Ambiental), cuyas etapas se describen en la figura 1 que se presenta a continuación.

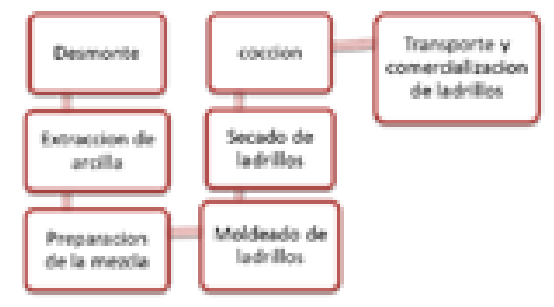

Figura 1. Proceso de extracción de arcilla elaboración de ladrillos en la mina Sabanilla. 
Las técnicas usadas en este estudio para la recolección de datos e información en primera instancia son la observación objetiva y empírica de las situaciones que se presentan en la unidad minera estudiada, la realización de una serie de entrevistas y encuestas a los distintos actores (tomando como principal referente al propietario de la mina.
La población involucrada en el presente estudio fueron los treinta trabajadores de la mina de arcilla Sabanilla del municipio San Juan del Cesar, La Guajira, así como los habitantes de la zona aledaña, correspondiente al barrio Loma Fresca, con una población aproximada de 140 individuos.

\section{Resultados}

Para esta revisión se tuvo en cuenta, principalmente, la entrevista realizada al encargado de la mina, la cual arrojó

como resultados más relevantes los datos que se presentan a continuación en la tabla 1.

Tabla 1. Resultados de la entrevista realizada al encargado de la mina

\begin{tabular}{|l|l|l|}
\hline $\begin{array}{l}\text { El yacimiento está siendo } \\
\text { explotado de manera artesa- } \\
\text { nal e ilegal, ya que no cuenta } \\
\text { con ninguno de los requisitos } \\
\text { que exige la ley. }\end{array}$ & $\begin{array}{l}\text { No implementa ninguna medida } \\
\text { de gestión ambiental que le per- } \\
\text { mita prevenir, mitigar, corregir } \\
\text { o compensar el daño causado al } \\
\text { ambiente }\end{array}$ & $\begin{array}{l}\text { Cuenta aproximadamente con } \\
\text { 30 trabajadores, los cuales no } \\
\text { utilizan ningún implemento de } \\
\text { seguridad, por lo cual están ex- } \\
\text { puestos a todo tipo de peligros. }\end{array}$ \\
\hline $\begin{array}{l}\text { No existe una política am- } \\
\text { biental con objetivos y metas } \\
\text { definidas. }\end{array}$ & $\begin{array}{l}\text { No utilizan energía eléctrica en } \\
\text { ninguna de las actividades que } \\
\text { se realizan en las instalaciones } \\
\text { de la mina. }\end{array}$ & $\begin{array}{l}\text { No cumple con las condiciones } \\
\text { mínimas sanitarias, ya que no } \\
\text { cuenta con acueducto y alcan- } \\
\text { tarillado. }\end{array}$ \\
$\begin{array}{l}\text { No se implementan progra- } \\
\text { mas ambientales }\end{array}$ & & \\
\hline
\end{tabular}

En La mina de arcilla Sabanilla, haciendo revisión de las prácticas de gestión ambiental, se pudo determinar que esta no cuenta con ningún tipo de gestión ambiental o prácticas que conlleven al mejoramiento del entorno entre los trabajadores y el ambiente.
La tabla 2 presenta los resultados de la entrevista aplicada al encargado de la mina, al igual que las observaciones anotadas en las diferentes visitas realizadas al área de estudio permitieron elaborar el siguiente diagnóstico, en el que se presenta la información obtenida de cada uno de los componentes ambientales. 


\section{Tabla 2. Diagnóstico ambiental de la mina}

\begin{tabular}{|c|c|c|c|}
\hline Flora & Fauna & Agua & Aire \\
\hline $\begin{array}{l}\text { Antes de que la } \\
\text { unidad minera } \\
\text { iniciara sus traba- } \\
\text { jos, dentro de esta } \\
\text { y sus alrededores } \\
\text { se encontraba una } \\
\text { gran cantidad de } \\
\text { especies vege- } \\
\text { tales constituida } \\
\text { por árboles, ar- } \\
\text { bustos, hierba y } \\
\text { matorrales; de las } \\
\text { cuales predom- } \\
\text { inaban especies } \\
\text { nativas como el } \\
\text { barquito morao', } \\
\text { la ceiba, el yaru- } \\
\text { mo, etc. En la } \\
\text { actualidad esta } \\
\text { riqueza de flora } \\
\text { ha presentado } \\
\text { una disminución } \\
\text { abismal, debi- } \\
\text { do al desmonte } \\
\text { que se realiza } \\
\text { como primer } \\
\text { paso para iniciar } \\
\text { la extracción de } \\
\text { la arcilla, el cual } \\
\text { consiste en retirar } \\
\text { toda la capa vege- } \\
\text { tal que se encuen- } \\
\text { tra en la superf- } \\
\text { cie del terreno a } \\
\text { intervenir. }\end{array}$ & $\begin{array}{l}\text { Debido a la deforestación } \\
\text { incontrolada en la zona, } \\
\text { las especies faunísticas } \\
\text { que habitan en las dife- } \\
\text { rentes especies vegeta- } \\
\text { les se ven obligadas a } \\
\text { emigrar de sus hábitats } \\
\text { naturales a otros lugares } \\
\text { en los que tal vez no en- } \\
\text { cuentren todas las condi- } \\
\text { ciones requeridas para su } \\
\text { supervivencia, pudiendo } \\
\text { generarse problemas en } \\
\text { su desarrollo, en su re- } \\
\text { producción e inclusive la } \\
\text { muerte. }\end{array}$ & $\begin{array}{l}\text { El recurso hídrico re- } \\
\text { querido en el proceso de } \\
\text { fabricación de ladrillos y } \\
\text { durante todo el proceso } \\
\text { productivo de la mina no } \\
\text { es muy elevado, general- } \\
\text { mente es tomado de los } \\
\text { hoyos que alcanzan una } \\
\text { profundidad mayor al ni- } \\
\text { vel freático, por lo cual se } \\
\text { llenan de agua subterrá- } \\
\text { nea o del agua lluvia que } \\
\text { se almacena en las cante- } \\
\text { ras. En ocasiones, cuan- } \\
\text { do no cuentan con estas } \\
\text { aguas naturales, el recur- } \\
\text { so requerido es tomado } \\
\text { del acueducto y trans- } \\
\text { portado con mangueras, } \\
\text { a partir las casas vecinas } \\
\text { hasta las instalaciones de } \\
\text { la mina. }\end{array}$ & $\begin{array}{l}\text { El aire en esta zona } \\
\text { se ve afectado de di- } \\
\text { ferentes formas; una } \\
\text { es por el polvillo que } \\
\text { se genera durante el } \\
\text { desprendimiento de } \\
\text { la arcilla del suelo, } \\
\text { teniendo en cuenta } \\
\text { que, por su tamaño, } \\
\text { las arcillas entran en } \\
\text { el grupo de coloides, } \\
\text { los cuales tienen la } \\
\text { capacidad de sus- } \\
\text { penderse en el aire } \\
\text { durante un tiempo } \\
\text { suficiente como para } \\
\text { ser consideradas un } \\
\text { contaminante en la } \\
\text { atmósfera. La otra } \\
\text { forma de contami- } \\
\text { nación atmosférica, } \\
\text { y la más importan- } \\
\text { te que ocurre en la } \\
\text { mina, es aquella } \\
\text { que se da debido al } \\
\text { uso de hornos para } \\
\text { la quema de los la- } \\
\text { drillos, los cuales } \\
\text { son alimentados con } \\
\text { leña y ACPM. }\end{array}$ \\
\hline
\end{tabular}




\begin{tabular}{|c|c|c|}
\hline Paisaje & Seguridad y salud & Generación de empleo \\
\hline $\begin{array}{l}\text { El paisaje de la zona también es } \\
\text { uno de los grandes afectados, en } \\
\text { él se pueden observar aspectos } \\
\text { ambientales altamente notorios } \\
\text { que, en conjunto, resultan en un } \\
\text { alto grado de degradación de la } \\
\text { estética del paisaje, como son la } \\
\text { pérdida de la vegetación, la alte- } \\
\text { ración de la topografía, y el mal } \\
\text { manejo de los residuos sólidos, } \\
\text { como trozos de ladrillos defec- } \\
\text { tuosos, llantas, palos, botellas y } \\
\text { toda clase de envolturas plásticas } \\
\text { de productos que utilizan los tra- } \\
\text { bajadores principalmente para su } \\
\text { alimentación. }\end{array}$ & $\begin{array}{l}\text { Es evidente que los traba- } \\
\text { jadores no cuentan con los } \\
\text { elementos ni la capacitación } \\
\text { necesaria para trabajar de ma- } \\
\text { nera segura, de tal forma que } \\
\text { se evite al máximo la ocurren- } \\
\text { cia de accidentes, dado que } \\
\text { laboran en condiciones inade- } \\
\text { cuadas, debido a que no utili- } \\
\text { zan uniforme, guantes, botas, } \\
\text { casco, etc., No existe un plan } \\
\text { de contingencias en caso de } \\
\text { emergencias y los trabajado- } \\
\text { res no cuentan con afiliación } \\
\text { a un seguro de salud. }\end{array}$ & $\begin{array}{l}\text { En este aspecto es importante } \\
\text { mencionar que la mina no perte- } \\
\text { nece a una sola persona como en } \\
\text { la mayoría de los casos, sino que } \\
\text { está subdividida y cada sección } \\
\text { tiene un propietario diferente sin } \\
\text { ningún tipo de sociedad u or- } \\
\text { ganización entre los mismos, y } \\
\text { cada uno está en la libertad de } \\
\text { contratar a los trabajadores que } \\
\text { considere necesario, en prome- } \\
\text { dio cada uno contrata entre tres a } \\
\text { cinco personas de escasos recur- } \\
\text { sos y, en su mayoría, con bajo } \\
\text { nivel de escolaridad, que aunque } \\
\text { en muchos casos no reciben un } \\
\text { salario adecuado, encuentran en } \\
\text { este empleo el sustento para sus } \\
\text { familias. }\end{array}$ \\
\hline
\end{tabular}

Básicamente estos factores ya mencionados son los que afectan de manera directa o indirecta, ya que, como se ha mencionado antes, la flora es afecta por la tala intensiva de árboles que se realiza en esta zona y, peor aún, no contribuyen con el reparo de este daño, ni quiera plantando un árbol, para así evitar que terminen por desaparecer dichas especies. A su vez, se encuentran en peligro de extinción ciertas especies que son nativas de ese mismo hábitat y que, por causa de la misma tala de árboles, han tenido que emigrar, ya que no encuentran ese espacio acorde con sus necesidades. En lo que respecta al aire se ve también afectado, ya que esas emisión de gases o, en su defecto humo, se concentra y, a su vez, se esparce cerca de las comunidades que se encuentran aledañas a estos sitios mineros y, desde luego, puede afectar a la salud humana. El suelo y el agua que ellos utilizan son también de esas mismas comunidades al momento de darle un uso no tienen la precaución de tomar o usar con cierto cuidado, simplemente van hasta el lugar en el que se encuentra el agua, la toman y listo, toda esta actividad no cuenta como tal con un reglamento que deban tener en cuenta para así disponer de los mismos recursos y espacios. 
En la siguiente tabla se presenta el contexto interno y externo de la mina de arcilla sabanilla, analizado a través de una matriz DOFA (fortalezas, debilidades, amenazas y oportunidades).

\section{Tabla 3. Matriz DOFA (fortalezas, debilidades, amenazas y oportunidades)}

\begin{tabular}{|c|c|}
\hline \multicolumn{2}{|c|}{ Matriz DOFA } \\
\hline Fortalezas (F) & Debilidades (D) \\
\hline $\begin{array}{l}\text { - Más de quince años de experiencia en la } \\
\text { producción de ladrillos. } \\
\text { - Aceptación de sus productos en el mer- } \\
\text { cado. } \\
\text { - Se maneja un precio competitivo del } \\
\text { producto. }\end{array}$ & $\begin{array}{l}\text { - La empresa no cuenta con ningún requisito } \\
\text { legal que exige la ley. } \\
\text { - No se implementa ninguna medida de gestión } \\
\text { ambiental. } \\
\text { - No está definida la política ambiental. } \\
\text { - } \quad \text { Falta de tecnologías adecuadas. } \\
\text { Carencia de infraestructura. }\end{array}$ \\
\hline
\end{tabular}

\begin{tabular}{|c|c|}
\hline Estrategias (FO) & Estrategias (DO) \\
\hline $\begin{array}{l}\text { Implementación de un sistema } \\
\text { de gestión integral con base en } \\
\text { las normas ISO 14001:2015. } \\
\text { Estudiar nuevos mercados para } \\
\text { incrementar el volumen de } \\
\text { venta del producto. } \\
\text { - Fomentar mayor interés de los } \\
\text { clientes hacia la mina. }\end{array}$ & $\begin{array}{l}\text { - Implementar sistemas eficientes que per- } \\
\text { mitan ejercer una revisión y un control } \\
\text { permanente de todos los procesos que se } \\
\text { implementan en la mina. } \\
\text { Desarrollar y promover la imagen de la } \\
\text { empresa frente al mercado. }\end{array}$ \\
\hline Estrategias (FA) & Estrategias (DA) \\
\hline $\begin{array}{l}\text { - Cumplir los requisitos legales } \\
\text { que exige la ley. } \\
\text { Aumentar la participación de } \\
\text { los integrantes de la mina en el } \\
\text { mejoramiento continuo y eficaz } \\
\text { de los estándares de seguridad, } \\
\text { salud ocupacional y cuidado del } \\
\text { medio ambiente al interior de la } \\
\text { organización. } \\
\text { Preparar estrategias de mercado } \\
\text { para impulsar la competencia } \\
\text { empresarial. }\end{array}$ & 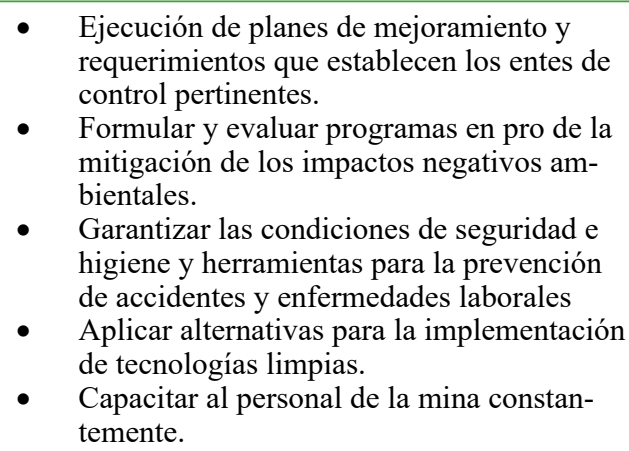 \\
\hline
\end{tabular}




\section{Conclusiones}

El análisis de los aspectos internos y externos asociados a la mina de arcilla Sabanilla contribuyó a la comprensión de su contexto, lo cual fue necesario para establecer un punto de partida sobre el que cimentar las bases para el diseño del Sistema de Gestión Ambiental. En este sentido, fue posible identificar las partes interesadas y sus necesidades y expectativas con respeto a la mina, logrando determinar que las principales son la comunidad, los empleados y los clientes. Se definió, además, que el SGA abarcará todos y cada uno de los componentes que forman parte de la red de procesos de la unidad minera, considerando también la totalidad de productos y actividades inmersos dentro de esta.

Así mismo, el análisis realizado arrojó como resultado que la mina no lleva a cabo ningún tipo de gestión ambiental, de ahí la necesidad de diseñar un sistema, con el fin de cumplir con los requisitos legales y las exigencias del mercado nacional, el cual, una vez implementado, mejorará el desempeño ambiental, control de costos, relaciones con las autoridades e industria, la reducción los incidentes ambientales, entre otros beneficios.
Durante la planificación del SGA, se logró la identificación de trece aspectos ambientales (cambios en el entorno paisajístico, pérdida de la cobertura vegetal, producción de residuos orgánicos como troncos, hojas, etc., generación de material particulado, remoción de suelo, carencia de elementos de protección personal, consumo de agua, generación de residuos por ladrillos defectuosos, emisión de gases contaminantes por combustión incompleta, uso de madera como combustible, falta de preparación de ante posibles incendios, producción de residuos sólidos domésticos por consumo de alimentos o refrigerios, ausencia de un lugar para la higiene de los empleados), los cuales son producto de todas las actividades que forman parte de la red de procesos de la mina.

Entre los hallazgos encontrados cabe mencionar el incumplimiento de la mayor parte de los requisitos legales exigidos por la normativa colombiana y que deben tenerse muy en cuenta para la implantación y el correcto funcionamiento del Sistema de Gestión Ambiental.

Así mismo, se determinaron concreta y sintetizadamente los objetivos ambientales que debe cumplir dicha unidad minera, cada uno encaminado hacia metas diferentes, pero con un fin común: 
"el manejo de los impactos ambientales generados por la actividad en la zona de influencia”. Para el logro de estos, se propusieron siete programas de gestión ambiental que corresponden al manejo de la contaminación atmosférica, los residuos sólidos, los escombros, los suelos, la fauna y flora, la seguridad y salud en el trabajo y la contratación de mano de obra.

\section{Referencias}

Acosta, A. (2014). Foro de Minería Responsable. Universidad de La Guajira. Riohacha, La Guajira.

Cardona, M.M., Chiner, S.E. y Lattur D.A. (2005). Diagnóstico psicopedagógico. Conceptos básicos y aplicaciones. San Vicente, Alicante: Club universitario. Recuperado de http://www.editorial-club-universitario.es/pdf/4412.pdf

Gaviria, L. (2007). Diagnóstico del estado actual de la gestión ambiental frente a la norma ISO 14001 en la empresa de acueducto y alcantarillado de Pereira. Pereira: Universidad tecnológica de Pereira.

Hernández, R. (2014). Metodología de la investigación. 6. ${ }^{\mathrm{a}}$ ed. Ciudad de México,
México: Mcgraw-Hill-Interamericana Editores.

Instituto Colombiano de Normas Técnicas y Certificación (Icontec). (2005) Implementar un sistema de gestión ambiental según ISO 14001: Guia básica para las empresas comprometidas con el futuro. Bogotá, D. C.

López, D. (2011). Mucho más carbón, el escenario minero de La Guajira. Riohacha: Universidad de la Guajira.

Ministerio de Fomento, Industria y Comercio (MIFIC) (2015). Sistema de gestión ambiental. Recuperado de http://www.mific. gob.ni/GESTIONAMBIENTAL/SISTEMADEGESTIONAMBIENTAL.aspx

Ministerio de Minas y Energía (2003). Glosario Minero. Bogotá, D. C.

Sánchez, J. (2001). El medio ambiente en Colombia: uso del territorio en Colombia. Instituto de Ciencias Naturales. Facultad de Ciencias. Instituto de Estudios Ambientales. Universidad Nacional de Colombia. Instituto de Hidrología, Meteorología y Estudios Ambientales, Ministerio del Medio Ambiente. República de Colombia.

Villamizar. (14 de septiembre del 2016). Actividad minera mundial: producción mundial de minerales. Recuperado de https://historiaybiografias.com/actividad_minera/. 